\title{
El peligro de ejercer periodismo en México. Análisis de la cobertura informativa del asesinato de Javier Valdez según el enfoque del peace journalism.
}

\section{The risks of practicing journalism in Mexico. Analysis of the coverage of the murder of Javier Valdez according to the approach of peace journalism.}

\author{
Maria Teresa Nicolás Gavilán ${ }^{1}$ \\ Recibido: 13-10-2017 - Aceptado: 05-01-2018 \\ DOI: https://doi.org/10.26441/RC17.1-2018-A5
}

\begin{abstract}
RESUMEN: La violencia hacia periodistas en México y en especial en el estado de Sinaloa, ha aumentado sus cifras a través de los años. ¿En este entorno es posible ejercer un periodismo de paz propuesto por Galtung (2002)? Más aún ¿se puede informar sobre la violencia ejercida contra los periodistas con el enfoque del peace journalism? En este artículo se analizó la cobertura informativa dada al asesinato del periodista Javier Valdez Cárdenas, hecho que revolucionó a periodistas y medios de comunicación de todo el país. A través del análisis de contenido (Berelson 1952; Krippendorf 1990) se revisaron los enfoques noticiosos de 366 noticias de los periódicos locales, en su versión en línea, Noroeste y El Debate, y de los nacionales Reforma y El Universal. Los resultados mostraron que, en general, la cobertura fue con un enfoque hacia el war journalism.
\end{abstract}

Palabras clave: periodismo de paz; periodistas; narcotráfico; impunidad; México.

ABSTRACT: Violence against journalists in Mexico and especially in the state of Sinaloa has increased its numbers over the years. In this environment is it possible to practice peace journalism proposed by Galtung (2002)? Furthermore, can one report on the violence exercised against journalists with the approach of peace journalism? In this article, the information coverage given to the murder of journalist Javier Valdez Cárdenas was analyzed, a fact that revolutionized journalists and media throughout the country. Through content analysis (Berelson 1952; Krippendorf 1990) the news approaches of 366 news from local newspapers, in its online version, Noroeste and El Debate, and from national ones Reforma and El Universal. The results showed that, in general, the coverage was with a focus towards war journalism.

Keywords: journalism; journalists; drug trafficking; impunity; Mexico.

1 María Teresa Nicolás Gavilán es profesora e investigadora de la Escuela de Comunicación de la Universidad Panamericana (México). Además es Vicerrectora de Humanidades del mismo centro. Es miembro del Sistema Nacional de Investigadores (SNI) en México. Chair del Working Group Ethics of Society and Ethics of Communication de la International Association of Media Communication Research (IAMCR). mnicolas@up.edu.mx, http://orcid. org/0000-0001-7385-8196 


\section{Introducción}

El riesgo que se corre como reportero en un entorno violento es muchas veces inimaginable para los mismos periodistas, hasta que se encuentran en la zona del conflicto y viven los enfrentamientos en carne propia.

En el contexto de un entorno y sociedad amenazados por el peligro constante, donde los índices de violencia se mantienen y suben, pero no bajan, el estado de Sinaloa representa un lugar complicado para ejercer la labor del periodismo. En la capital de este estado Culiacán- fue asesinado el periodista Javier Valdez el 15 de mayo del 2017; fue el sexto periodista asesinado en el año 2017, que concluye con 12 reporteros que han perdido la vida como víctimas de la violencia.

En este artículo se analiza la cobertura periodística dada a este suceso en los periódicos mexicanos locales y nacionales a la luz de las propuestas del periodismo de paz o peace journalism.

\subsection{El ejercicio del periodismo en México. La amenaza del Narcotráfico.}

En un estudio realizado por Márquez-Ramírez (2016) se mostraron varios datos relevantes del ejercicio del periodismo en México: destacan las condiciones de precariedad de los periodistas que tienen bajos salarios, mucha carga de trabajo y pluriempleo. El estudio muestra que los periodistas mexicanos sufren una amplia gama de presiones y se enfrentan a situaciones y desafíos todos los días.

Estas presiones se pueden agrupar en tres: la política, la económica y la vio- lencia contra los periodistas.

El sistema político tuvo gran influencia sobre los medios durante muchos años, prácticamente los periodistas adulaban y publicaban noticias en complicidad con el poder. Con este contexto, era de esperarse una baja autonomía periodística y autocensura automática, resultados de la influencia que sostuvo el poder político en los medios (Monsiváis, 2003).

Estrechamente unido al anterior se encuentra el factor económico, ya que como expone The New York Times, el dinero que destina el gobierno mexicano al pago de espacios de publicidad en los medios es:

una de las restricciones más severas a la libertad de expresión que enfrentan los medios de comunicación, provocando que reporteros y editores sean a menudo sometidos a la influencia del gobierno, que periodistas abiertamente críticos sean despedidos, que historias negativas sean censuradas y que informes de investigación sean frustrados (New York Times, 2017).

Según este diario, en México el 38\% del gasto hecho en 2016 en publicidad televisiva y más del $16 \%$ de la publicidad en radio, proviene del gobierno.

El tercer factor de presión es la violencia contra los periodistas. En el último informe de la UNESCO "Tendencias mundiales en Libertad de Expresión y Desarrollo. Informe mundial 2017-2018" se muestra que México es el tercer país a nivel mundial con mayor número de asesinatos de periodistas en el periodo 2012-2016, por debajo de la República Árabe de Siria y de 
Irak. En cuanto a América Latina es el número uno (Figura 1). El año 2017 fue particularmente nocivo para los medios ya que entre los meses de marzo y diciembre en México han sido asesinados 12 periodistas ${ }^{2}$.

Figura 1. Número de periodistas asesinados por país, 2012-2016

\section{Número de periodistas asesinados por país, 2012-2016}
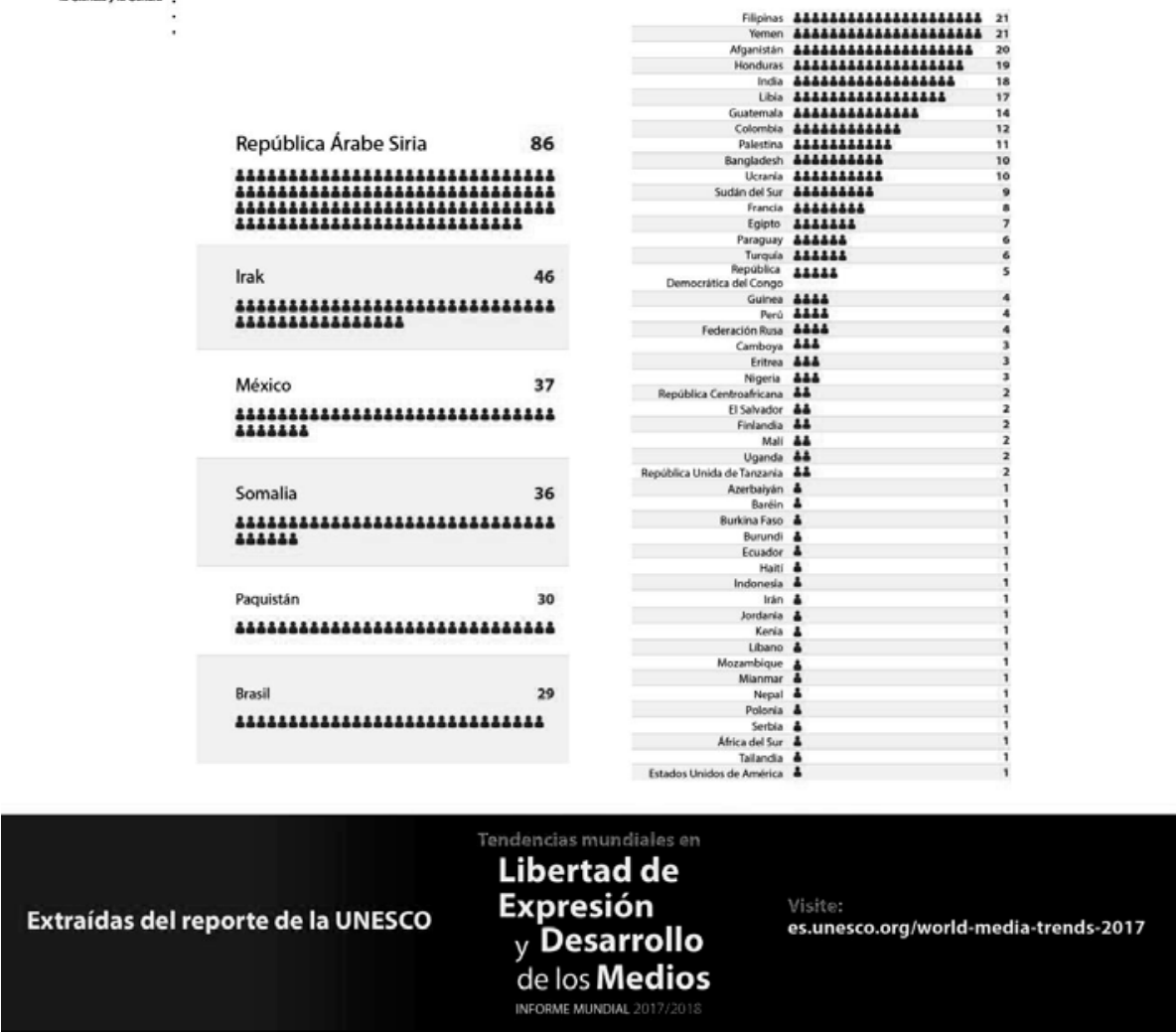

2 Los periodistas asesinados son: Cecilio Pineda, Ricardo Monlui, Miroslava Breach, Máximo Rodríguez, Javier Valdez, Jonathan Rodriguez Córdoba, Salvador Adame, Edwin Rivera, Luciano Rivera, Cándido Ríos, Edgar Daniel Esqueda y Gumaro Pérez Aguilando Cfr. Gatopardo 2017 https://www.gatopardo.com/opinion/actualidad/ periodistas-asesinados-en-mexico-en-2017/ 
La edición 2017 de la Clasificación Mundial de Libertad de Prensa de Reporteros sin Fronteras incluyó a 180 países, de los cuales México y Cuba presentaron las peores condiciones para los periodistas de Latinoamérica (en los lugares 147 y 173 de la lista, respectivamente). Según este reporte, los países nórdicos no poseen riesgo alguno para practicar el periodismo; Estados Unidos, Canadá y Australia son considerados un buen lugar, sin embargo, México es posicionado en el nivel denominado "situación difícil", siendo uno de los países con menor libertad de expresión en América Latina.

El Committee to Protect Journalists (CPJ 2017a) clasifica a los asesinos de periodistas a nivel mundial en seis categorías: gobiernos, militares, paramilitares, grupos políticos, muchedumbres y residentes locales.

En este sentido Meneses (2011) afirma que hay muchas otras situaciones por las cuáles ejercer esa labor es sinónimo de peligro. La mayoría de los abusos que se cometen contra periodistas son realizados por funcionarios públicos o personas que se encuentran en el poder. Sin embargo, en los últimos años podemos comprobar que la rivalidad delincuencial es, en gran medida, la responsable de que los grupos y cárteles del crimen organizado atenten contra la vida de reporteros.

Un dato relevante es observar la tasa promedio de homicidios por rivalidad delincuencial en municipios. En los que se ha asesinado a periodistas es de $20.29 \%$, por el contrario, en donde no ha habido víctimas de este tipo, el por- centaje es de 9,2\% (Ríos, 2012). También es importante considerar que entre los Municipios más violentos se encuentran: Guadalupe, Mérida y General Treviño, ahí las tasas de homicidio por rivalidad delincuencial alcanzan cifras de 538.07, 490.50 y 331.23 casos anuales por 100 mil habitantes respectivamente. Otros municipios que sufren este tipo de violencia son Culiacán con 2345 casos, Tijuana 1895 casos y Mazatlán con 739 casos de 2007 a 2011 (Ríos, 2012).

En 2010, El Diario de Ciudad Juárez publicó en su editorial un comunicado dirigido en primera parte a las "diferentes organizaciones que se disputan la plaza" y, en segundo lugar, a las autoridades que no han hecho su trabajo por representar la justicia en ese lugar. Este editorial fue un parteaguas en el periodismo nacional, ya que un periódico importante alzó la voz y señaló que el Estado Mexicano estaba ausente ( $E l$ Universal, 2010).

Señores de las diferentes organizaciones que se disputan la plaza de Ciudad Juárez: la pérdida de dos reporteros de esta casa editora en menos de dos años representa un quebranto irreparable para todos los que laboramos aquí y, en particular, para sus familias. Hacemos de su conocimiento que somos comunicadores, no adivinos. Por tanto, como trabajadores de la información queremos que nos expliquen qué es lo que quieren de nosotros, qué es lo que pretenden que publiquemos o dejemos de publicar, para saber a qué atenernos (El Diario de Ciudad Juárez, 2010). 
Salazar (2012), señala en un artículo varios testimonios de periodistas de Ciudad Juárez, que hablan sobre su ejercicio en esta zona de riesgo. Un testimonio muestra que lo que sintieron después de los distintos ataques a El Diario, y cómo entre su grupo de trabajo hablaron y decidieron enfrentar la situación con la mayor entrega y compromiso colectivo que les fuera posibles, afirmando que no guardaran silencio (Entrevista a periodista 5).

A través de los años, el índice de muerte de periodistas ha aumentado, principalmente desde que los líderes del narcotráfico decidieron que México era una mejor base para operar que Colombia, y que esta última, debía quedarse como productor de materia prima. Se puede observar una relación inversamente proporcional entre estos dos países, de 1997 a 2003, Colombia experimentó cerca de 9 asesinatos de periodistas y México menos de uno. "De 2004 a 2010 México se ha convertido en el país más peligroso para el ejercicio del periodismo en toda Latinoamérica” (Ríos, 2012).

En Sinaloa se realizaron cinco agresiones a periodistas en 2015 y nueve en el 2016. Incluso, el único medio estatal que aparece en la lista de los medios con más agresiones en 2016 es Noroeste, periódico local sinaloense.

Aunado a lo anterior, se da el hecho de que muchos de los crímenes contra los periodistas quedan impunes. Según muestra en su página web el Committee to Protect Journalists en Mexico desde 1992 a 2017 han sido asesinados 43 periodistas, 40 de esos fueron blanco de asesinato y 35 de esos fueron crímenes ha permanecido impunes (CPJ, 2017b). Recordemos que entre los meses de marzo y diciembre de 2017 en México han sido asesinados 12 periodistas y que todos estos crímenes siguen sin tener una resolución judicial (Gatopardo, 2017).

Todo lo anterior nos lleva a la siguiente interrogante, ¿en este panorama complejo se puede ejercer un periodismo que contribuya a la paz?

\section{El modelo del Peace Journalism}

Los políticos, los grupos de poder y los científicos sociales, señalan el papel primordial que tienen los medios de comunicación en la cobertura de los conflictos sociales, políticos y bélicos (Galtung and Vincent, 1992; Roach, 1993; Shinar, 2003, 2004; Lee et al., 2006; Kuusik, 2010; Gavra y Savitskaya, 2011). La batalla sobre los medios de comunicación es uno de los grandes elementos en los conflictos modernos. Cada antagonista trata de promover su propio enfoque del conflicto en los medios de comunicación como un intento de movilizar un soporte político para su causa (Wolfsfeld, 1997, 2004).

Johan Galtung -pionero en los estudios sobre la paz ${ }^{3}$ - analizó el rol que tienen los medios de comunicación en

3 Johan Galtung es considerado el fundador de esta disciplina. En 1959 creó el Peace Research Institute, cinco años más tarde fundó el Journal of Peace Research (1964) y, en 2000, el Nordic Institute for Peace Research. 
los conflictos. Como fruto de sus estudios, afirmó que los medios de comunicación si bien no generan la violencia, sí contribuyen a acentuarla a través de ciertas orientaciones lingüísticas y de los temas en los que centran su atención al cubrir una información. Galtung consideró que los periodistas tenían el deber ético de contribuir a la resolución de los conflictos (Galtung, 2006).

Asimismo, él consideraba que hay dos modos de cubrir un conflicto: el camino bajo (low road) y el camino alto (high road). Cada camino difiere por su enfoque. El primero se fija en el aspecto conflictivo, mientras que el segundo se orienta hacia la transformación pacífica del conflicto estudiando sus raíces (Galtung, 1997, 1998b)

El low road es el llamado "periodismo de guerra" o war journalism. Este periodismo presenta cuatro características principales: está orientado hacia la violencia y la guerra, está muy influido por la propaganda, presenta la opinión de las élites y concibe la paz como la victoria de unos sobre otros. De igual manera, aborda el conflicto como una batalla, un deporte, o un círculo de gladiadores, es decir, las crónicas se refieren a quién avanza, quién se rinde y quién ha sufrido más muertos o daños. Por lo tanto, su enfoque es llamado "zero-suma game", o sea, una parte gana todo y la otra lo pierde todo (Galtung, 2002).

Por el contrario, el "Periodismo de Paz" o Peace Journalism se orienta hacia la transformación del conflicto, busca reflejar la verdad, atiende la opinión de todas las personas involucradas y entiende la paz como la solución de un conflicto, donde todas las partes implicadas reciben un beneficio (Galtung, 1998b). Estas actitudes se pueden sintetizar en cuatro propuestas normativas: (1) El periodista debe hacer un análisis del conflicto para poder informar sobre hechos violentos, es decir, conocer los orígenes y las causas, así como quiénes son las partes contendientes, y qué objetivos tienen. (2) La información debe presentar una orientación hacia las soluciones del conflicto, notificando, por ejemplo, las propuestas, las negociaciones, los acuerdos, etcétera. (3) El periodista debe perseguir la verdad simétrica, es decir, la realidad -positiva y negativade las partes contendientes, no sólo de una de ellas; evitar, por lo tanto, presentar a las partes de una manera maniquea, como buenos y malos y/o víctimas y verdugos. (4) La orientación de una noticia debe estar dirigida hacia la gente común y no sólo hacia las élites; de manera que el informador debe atender tanto las ruedas de prensa de cancilleres y políticos como al público en general, dando voz a todos los actores que intervienen o son afectados en un conflicto.

Galtung presenta no sólo un planteamiento normativo, sino que también propone orientaciones concretas para analizar y elaborar las noticias, según los criterios del Peace Journalism. Por ello, investigadores sobre el gran tema de la paz, plantean el Peace Journalism como un modelo a través del cual los medios pueden ayudar a 
los ciudadanos a tomar decisiones que favorezcan la paz (Shinar, 2004). Para contribuir a la misma, este modelo presenta cuatro aspectos fundamentales -tanto de fondo y forma-: en primer lugar, considerar que todas las noticias tienen un enfoque o frame informativo determinado, proponiendo que éste sea para la paz -peace frame(Nicolas-Gavilan, 2011); en segundo lugar, utilizar la herramienta del análisis del conflicto para entender la situación de la que informan (Galtung, 1998a); el tercer punto es construir las noticias siguiendo ciertas pautas determinadas (Galtung, 2002), y, en cuarto lugar, considerar que los periodistas tienen la responsabilidad social de tomar una "opción ética" en su trabajo y por lo tanto, contribuir a la paz (Galtung, 2006).

Al respecto, Galtung $(1997 ; 1998 a)$ y Lynch (2013) presentan no sólo un planteamiento normativo, sino que también proponen orientaciones concretas para analizar y elaborar las noticias según los criterios del Peace Journalism.

Con base en las propuestas del Peace Journalism (Galtung, 2002; Lynch, 2015) y la experiencia de la autora en la aplicación de este modelo para analizar conflictos (Nicolas-Gavilan, 2012, y 2015), se proponen siete características -que se pueden considerar categorías de análisis de contenido- para determinar si una noticia tiene un enfoque de Peace Journalism o War Journalism. (1) La noticia debe mostrar los antecedentes y el contexto de la formación del conflicto. (2) La nota debe presentar diversas opiniones de cada bando enfrentado o afectado, en otras palabras, debe darle voz a todos los puntos de vista de todos los partidos rivales. (3) Ofrecer ideas creativas para la resolución del conflicto, dando cierto protagonismo a los actores que proponen soluciones. (4) La información debe presentar a la sociedad civil como activa, no como simple agente pasivo que sufre las consecuencias de los enfrentamientos. (5) Las noticias tienen que favorecer la aparición de las propuestas de paz de los distintos actores y señalar los puntos positivos, dando seguimiento a la etapa de postconflicto. Estos cinco aspectos corresponden al contenido y fondo de la noticia, pero también es importante atender a la forma, es decir al cómo se redactan las noticias, en ese sentido proponemos analizar (6) el lenguaje para determinar si es racional o emotivo, es decir que no aporta datos sino emociones. (7) Por último analizar si es un lenguaje neutro o culpabilizado, es decir que califica a los actores como buenos y malos.

Los puntos antes expuestos anterior se puede resumir en el siguiente cuadro: 
Cuadro 1. Categorías de Análisis de Contenido, con enfoque "Peace Journalism"

\begin{tabular}{|l|l|}
\hline $\begin{array}{l}\text { 1. ANTECEDENTES/ } \\
\text { BACKGROUND (BKGD) }\end{array}$ & $\begin{array}{l}\text { Explorar antecedentes y el contexto de la formación del } \\
\text { conflicto, presentar causas y las opciones de cada lado } \\
\text { para representar el conflicto en términos realistas y } \\
\text { transparentes para la audiencia. }\end{array}$ \\
\hline $\begin{array}{l}\text { 2. PUNTOS DE VISTA/ } \\
\text { VIEWS } \\
\text { (VIEWS) }\end{array}$ & $\begin{array}{l}\text { Darle voz a todos los puntos de vista de todos los partidos } \\
\text { rivales. }\end{array}$ \\
\hline 3. IDEAS/IDEA (IDEA) & $\begin{array}{l}\text { Ofrecer ideas creativas para la resolución del conflicto, } \\
\text { desarrollo, pacificación y preservación de la paz. }\end{array}$ \\
\hline $\begin{array}{l}\text { 4. PROACTIVIDAD/ } \\
\text { PROACTIVE (PRO) }\end{array}$ & $\begin{array}{l}\text { Presentar a la sociedad civil como activa, no como simple } \\
\text { agente pasivo }\end{array}$ \\
\hline $\begin{array}{l}\text { 5. PRO-PAZIPEACE } \\
\text { (PEACE) }\end{array}$ & $\begin{array}{l}\text { Poner atención a las historias de paz y el desarrollo } \\
\text { postguerra. }\end{array}$ \\
\hline $\begin{array}{l}\text { 6. LENGUAJE EMOTIVO/ } \\
\text { EMOTIVE (EMOT) }\end{array}$ & $\begin{array}{l}\text { Utilizar un lenguaje racional con datos y evitar usar sólo un } \\
\text { lenguaje emotivo. }\end{array}$ \\
\hline $\begin{array}{l}\text { 7. ETIQUETAR/LABEL } \\
\text { (LABEL) }\end{array}$ & $\begin{array}{l}\text { Evitar etiquetar a las partes clasificando a unos de buenos } \\
\text { y otros de malos }\end{array}$ \\
\hline
\end{tabular}

Fuente: Lynch 2013, Nicolas-Gavilan (2012, 2015). Elaboración Propia.

La teoría del periodismo de paz se ha desarrollado especialmente durante los últimos 20 años Sus impulsores han llevado a cabo mucho trabajo en enseñar cómo aplicar el periodismo de paz.

Existen estudios generales sobre el periodismo de paz en México, por ejemplo, los de Cristina Ávila-Zesatti (2015). Además, hay otros estudios especializados en el análisis de las noticias sobre el narcotráfico en México a la luz de las propuestas del Peace Journalism (Nicolas-Gavilan, 2012; Lynch, 2013; Cottle, 2016; Howard Tumber and Waisbord, 2017). Sin embargo, no se ha estudiado la cobertura informativa sobre la muerte de los periodistas a manos de los grupos narcotraficantes, por lo que este artículo puede ser una aportación interesante.
3. Caso de estudio: la violencia en Culiacán y el asesinato de Javier Valdez.

Culiacán, capital del estado de Sinaloa, es muy famosa a nivel mundial por sus traficantes de narcóticos y por poseer los cárteles más poderosos de todos los tiempos. La situación de violencia y tráfico de mercancías ilegales no es cosa del siglo XXI, esto viene de años y años de trayectoria delictiva.

El presidente José López Portillo, presionado por el gobierno de Estados Unidos, puso en marcha la Operación Cóndor en el año de 1977, que consistía en erradicar los cultivos ilícitos, capturar narcotraficantes y restaurar la seguridad en el estado de Sinaloa. Fue la primera vez que el ejército mexicano se involucró en este tipo de operaciones, 
ocasionando que los narcos se salieran de Tierra Blanca, donde se concentraban la mayoría de los capos sinaloenses más famosos ${ }^{4}$. Los narcos se esparcieron por todo Sinaloa, otros estados de México y hasta en el extranjero. El éxodo facilitó, de hecho, a que los capos hicieran y abrieran su mercado a otros lugares, lo cual podría llamarse efecto cucaracha.

A través de los años, el Cártel de Sinaloa fue ganando poder, sobre todo desde que Joaquín Guzmán Loera, quien hace poco era el hombre más buscado del mundo, tomó el liderazgo del grupo delictivo. El Cartel de Sinaloa es el más representativo de México, y es también el medio por el cual se trafica la droga, desde su producción en Colombia, hasta su distribución en Estados Unidos.

Debido a este entorno, el periodista sinaloense se encuentra envuelto en un ambiente de peligro, y esto no es nuevo, sirva como ejemplo señalar que hace más de 10 años el estado de Sinaloa fue la zona de la República Mexicana con mayor número de homicidios dolosos cometidos durante ajustes de cuentas de las bandas del crimen organizado (López, 2008). Siguiendo con la revisión histórica de la violencia contra periodistas y presidentes muni- cipales de los estados mexicanos, Sinaloa cuenta con 4 muertes a periodistas dentro del periodo de 1994 a 2011, mientras que no hay ninguna muerte registrada de presidentes municipales (Ríos, 2012).

El 15 de mayo 2017, a plena luz, fue asesinado el periodista Javier Valdez Cárdenas ${ }^{5}$.

Este periodista era respetado y reconocido por las publicaciones que hizo sobre temas del crimen organizado, la mayoría de los periodistas que cubrían notas en Sinaloa le solicitaban información a Javier. Fue autor de varios libros como Miss Narco (2007), Los morros del narco (2007), Malayerba (2010) -que es también el nombre de su columna en Río doce-, Con una granada en la boca (2014), Huérfanos del narco (2015), y Narco periodismo (2016).

Éste último trató sobre el peligro que corren las personas que ejercen la profesión del periodismo, así como también la prensa en medio del crimen, la censura y la denuncia. En su libro se dedicó a entrevistar periodistas de todas las zonas de alto riesgo del país, para que ellos desde el anonimato o dando nombres, contaran sus experiencias personales y su sentimiento al cubrir información relacionada con delincuencia organizada.

4 Tierra Blanca es una colonia de Culiacán muy reconocida porque ahí, anteriormente, era la cuna de los mafiosos de la zona; cualquier persona que viviera o pasara por ahí era mal vista.

5 Javier Valdez estudió sociología en la Universidad Autónoma de Sinaloa, sus inicios como reportero fueron en noticieros televisivos del Canal 3, posteriormente ingresó a las páginas de Noroeste y desde 1998 se convirtió en corresponsal de La Jornada. Junto con un grupo de periodistas, en 2003 fundó el semanario Ríodoce, el cual es uno de los medios predilectos para informarse sobre temas del narcotráfico. Ríodoce fue víctima de un atentado en el 2009, se detonó una granada en la planta baja de las instalaciones del semanario, en la colonia Jorge Almada, en Culiacán. 
El contexto en el cual se gestó la muerte de Javier Valdez fue el siguiente: después de un periodo de relativa "paz" entre los grupos del crimen organizado en Sinaloa, re-aprehendieron al líder del Cártel de Sinaloa, "El Chapo" Guzmán. Eso desató una nueva ola de violencia en el estado, ya que sus hijos y Dámaso López Núñez alias "El Licenciado"- amigo y compañero de El Chapo- comenzaron a disputarse el liderazgo de la organización. Dámaso buscó espacio en los impresos de Sinaloa Río doce y La Pared, a quienes concedió entrevista vía telefónica y se deslinda de ataques que el hijo del Chapo le había atribuido. La entrevista la realizó Javier, porque lo buscaron específicamente a él. Los Chapitos se enteraron de la entrevista y presionaron a Javier para que no la publicaran, y una vez que fue negada la petición, le hablaron para comprar toda la edición, lo cual también se les negó. El 19 de febrero decidieron después perseguir al personal de entrega, esperar a que los dejaran en tiendas, y de esa manera comprarlos enseguida. El periódico y Javier comenzaron a sentir inseguridad, por lo que acordaron que el periodista se fuera a cubrir noticias en otros estados, pero eso tampoco se efectúo.

Como nos equivocamos también al entrevistar a Dámaso, porque de esa forma nos metimos a una guerra mediática que no era nuestra, provocando el disgusto - sin que fuera nuestra intención-de la otra parte. Por eso el decomiso de los ejemplares del domingo 19. (Bojórquez, 2017)

Javier había recibido amenazas tres semanas antes de su atentado:

En semanas recientes recibió amenazas de un calibre diferente al acostumbrado; él y su esposa Griselda realmente se preocuparon. Viajó a la Ciudad de México para consultar con los directivos de este periódico y con el Comité de Protección para Periodistas, con el que tenía lazos estrechos, sobre lo que procedía hacer (La Jornada, mayo 16 2017).

Después de eso, le recomendaron salir del país por su propia seguridad, pero no lo hizo.

El 15 de mayo del 2017, Río doce lanzó una escueta nota que decía:

El periodista de Río doce, Javier Valdez Cárdenas, fue asesinado hace unos momentos por la Calle Vicente Riva Palacio, entre Ramón F. Iturbe y Epitacio Osuna, de la colonia Jorge Almada. De acuerdo a los primeros datos, el también autor de la columna Malayerba, conducía un Corolla rojo de modelo reciente, por dicha vialidad, cuando sujetos lo interceptaron y le dispararon.

La primera versión oficial de los hechos decía que Javier Valdez fue atacado por sujetos encapuchados a unos metros de las instalaciones de Río doce. Valdez abordó su automóvil, y cuando se disponía a partir, los sicarios lo sorprendieron, lo obligaron a bajar del vehículo y le dispararon en repetidas ocasiones en la calle.

En el lugar de los hechos quedaron 12 casquillos de pistolas de 9 milímetros y 38 súper. Los agresores se llevaron el automóvil y alrededor de las 12:35 horas, la policía lo encontró cer- 
ca del sitio del crimen, en la calle Aquiles Serdán.

El periodista había recibido amenazas de muerte, tres meses anteriores a su asesinato, de manera anónima. La Procuraduría General de la República (PGR) anunció durante las horas posteriores a su muerte, que la principal línea de investigación es la actividad profesional de Javier Valdez. En un comunicado, la Fiscalía General del Estado (FGE) señaló que inició una investigación en coordinación con la PGR, por conducto de la Fiscalía Especial para la Atención de Delitos cometidos contra la Libertad de Expresión (Feadle).

Las teorías principales del asesinato están obviamente relacionadas con el crimen organizado. Sin embargo, a finales de mayo comenzaron a rondar teorías alternativas sobre el atentado. Una de ellas es desviar la atención de otros casos que hasta antes del asesinato de Valdez estaban en la mente de muchos sinaloenses, por ejemplo: las investigaciones y castigo de los responsables de saquear el Estado de Sinaloa.

Marchas, protestas, peticiones a las autoridades, entre otras manifestaciones siguen presentes después del asesinato del periodista Javier Valdez. La investigación sigue abierta.

\section{Objetivo de investigación}

Como ya se mencionó antes, existen algunas publicaciones que analizan la cobertura informativa sobre el narcotráfico en México desde la perspectiva teoría del peace journalism. Sin embargo, no hay estudios académicos que, a partir de las propuestas del peace journalism, analicen la cobertura informativa que dan los periódicos mexicanos a los asesinatos de los periodistas y las posteriores acciones que se derivan de estos.

El caso elegido para estudiar este tema es el asesinato del periodista Javier Valdez, que ha tenido un alto impacto mediático en el año 2017. El hecho fue cubierto tanto por prensa internacional, nacional y local. Se considera oportuno estudiar la información publicada por cuatro de los medios mexicanos -nacionales y locales- con impacto $\mathrm{y}$ alcance.

Formulación de la hipótesis:

Los medios locales son más propios para realizar una cobertura más amplia del suceso que ocurrió en su localidad y aplicar el enfoque del Peace Journalism, porque dan mayor contexto (BKGD).

El contexto en el que se da este hecho es el de una sociedad mexicana que ha cobrado más conciencia de su papel como fuerza social y exige a la autoridad que los asesinatos de periodistas no queden impunes. Así lo muestran las numerosas marchas, protestas, peticiones a las autoridades, etc., posteriores a la muerte del reportero y en las cuales se exigió la resolución del crimen, el castigo a los culpables y la futura protección a los periodistas en el ejercicio de su profesión.

Objetivo de investigación:

Realizar un análisis comparativo de la cobertura informativa del asesinato de Javier Valdez realizada por cuatro periódicos mexicanos -El Universal, Reforma, El Debate y Noroeste- en términos de las propuestas del Peace Journalism. 


\section{Metodología de investigación y muestra.}

Para realizar esta investigación se utiliza el análisis de contenido que es una técnica de interpretación de textos, en un sentido amplio.

El análisis de contenido, como técnica de investigación, se comenzó a utilizar a principios del siglo pasado, siendo H. Laswell su principal impulsor con sus estudios sobre la propaganda. Desde medios del siglo XX se define esta metodología, anotando sus elementos constitutivos. Para Berselon es "una técnica de investigación para la descripción objetiva, sistemática y cuantitativa del contenido manifiesto de comunicación" (1952: 18).

Esta definición implica -como señala el autor- ciertas reglas. La objetividad hace referencia a la utilización de procedimientos que puedan ser replicados por otros investigadores para verificar los resultados. La sistematización tiene también este fin, como señala Krippendorf (1990). Por lo que se refiere a las inferencias, se debe asumir como reales las inferencias sobre la relación entre la intención y el contenido o entre el contenido y el efecto; además se debe considerar que el estudio del contenido manifiesto es significativo (Berelson, 1952: 18,19 y 20).

Hostil (1969: 5) propone otra definición de análisis de contenido señalando que es "una técnica de investigación para formular inferencias identificando de manera sistemática y objetiva ciertas características específicas dentro de un texto". En esta definición se eliminan conceptos como contenidos cuantitati- vos y manifiestos, lo que amplia esta técnica para que sus análisis sean cualitativos y hagan referencia a contenidos latentes de los textos.

Por su parte, Krippendorff (1990: 28) señala que el análisis de contenido es "una técnica de investigación destinada a formular, a partir de ciertos datos, inferencias reproducibles y validas que puedan aplicarse a su contexto". Este último elemento -el contexto- es propuesto como el marco de referencia donde se desarrollan los mensajes y los significados

A partir de estas definiciones, unos años más tarde, Laurence Bardin (1996: 32) propone una definición general que abarca los elementos propuestos por sus antecesores, él define el análisis de contenido como "el conjunto de técnicas de análisis de las comunicaciones tendentes a obtener indicadores (cuantitativos o no) por procedimientos sistemáticos y objetivos de descripción del contenido de los mensajes permitiendo la inferencia de conocimientos relativos a las condiciones de producción/recepción (contexto social) de estos mensajes"

En consecuencia, con la aplicación de esta metodología de investigación se han seguido los distintos elementos y pasos que pide el proceso.

En primer lugar, se determinó el objetivo de análisis. La presente investigación, a través del análisis de contenido, busca estudiar y describir si se aplicó el enfoque propuesto del Peace Journalism en la cobertura del asesinato del periodista Javier Valdez en Culiacán dentro del contexto concreto en que estos hechos sucedieron. 
La imposibilidad de abarcar el universo de noticias publicadas sobre el asesinato de Javier Valdez obliga a determinar una unidad de muestro. La muestra está constituida por los textos informativos, interpretativos y de opinión, publicados en la versión digital de cuatro diarios, dos nacionales y dos medios locales: El Universal, Reforma, El Debate y Noroeste. El periodo de análisis abarca tres meses: comenzando la semana del 15 de mayo hasta la semana del 16 de julio del 2017. La elección de estos diarios mexicanos se debe a que los dos primeros son los diarios de mayor difusión y posicionamiento a nivel nacional; por su parte el tercero y el cuarto tienen buena difusión y posicionamiento en el Estado de Sinaloa. Rio doce se excluyó expresamente porque es el periódico fundado por Javier Valdez. Las fechas elegidas para el análisis corresponden a la cobertura inmediata tras el asesinato del periodista Javier Valdez.

La unidad de registro es todo texto temático impreso en cada diario. Se examinan todos los textos publicados por los periódicos seleccionados, ya sean informativos, interpretativos o de opinión. Con base en lo anterior, se recopilaron y analizaron 366 unidades periodísticas relacionadas con el asesinato de Valdez.
Para realizar el análisis de contenido se elaboró un programa de codificación con una serie de categorías que permitan dar respuesta al objetivo dispuesto. El sistema de codificación presenta dos niveles: uno de registro y clasificación de variables y otro de análisis cuantitativo.

Las variables del programa de codificación son de dos tipos: en primer lugar, los datos de registro de los textos -el nombre del periódico, el titular, el autor, la extensión y la fecha de publicación- en segundo lugar, las variables que se refieren al Peace Journalism.

De las categorías del peace journalism antes presentadas se han elegido 5 sólo excluyéndose expresamente dos que no aplican a la presenta investigación: (VIEWS) darle voz a todos los puntos de vista de todos los partidos rivales, $\mathrm{y}$ (PEACE) presentar las propuestas de paz de los distintos actores y señalar los puntos positivos, dando seguimiento a la etapa de post-conflicto. El objetivo de esta investigación -el asesinato del periodista Javier Valdez- es particular dentro de un conflicto mayor que es el crimen organizado en México y por lo tanto no se analizaron soluciones o propuestas de paz para este conflicto. Por lo tanto, analizaremos las siguientes categorías. 
Cuadro 2. Categorías de Análisis de Contenido seleccionadas.

\begin{tabular}{|l|l|}
\hline $\begin{array}{l}\text { ANTECEDENTES/ } \\
\text { BACKGROUND } \\
\text { (BKGD) }\end{array}$ & $\begin{array}{l}\text { Explorar antecedentes y el contexto de la formación del conflicto, } \\
\text { presentar causas y las opciones de cada lado para representar el } \\
\text { conflicto en términos realistas y transparentes para la audiencia. }\end{array}$ \\
\hline IDEAS/IDEA (IDEA) & $\begin{array}{l}\text { Ofrecer ideas creativas para la resolución del conflicto, } \\
\text { desarrollo, pacificación y preservación de la paz. }\end{array}$ \\
\hline $\begin{array}{l}\text { PROACTIVIDAD/ } \\
\text { PROACTIVE (PRO) }\end{array}$ & $\begin{array}{l}\text { Presentar a la sociedad civil como activa, no como simple agente } \\
\text { pasivo }\end{array}$ \\
\hline $\begin{array}{l}\text { LENGUAJE EMOTIVO/ / } \\
\text { EMOTIVE (EMOT) }\end{array}$ & $\begin{array}{l}\text { Utilizar un lenguaje racional con datos y evitar usar sólo un } \\
\text { lenguaje emotivo. }\end{array}$ \\
\hline $\begin{array}{l}\text { ETIQUETAR/LABEL } \\
\text { (LABEL) }\end{array}$ & $\begin{array}{l}\text { Evitar etiquetar a las partes clasificando a unos de buenos y otros } \\
\text { de malos }\end{array}$ \\
\hline
\end{tabular}

Fuente: Lynch 2013, Nicolas-Gavilán (2012, 2015). Elaboración Propia.

En el análisis de las noticias participaron dos codificadores con estudios universitarios $^{6}$. Se comprobó la fiabilidad de los codificadores.

Finalmente, la información obtenida fue procesada en Excel para la elaboración de las tablas y gráficos, las cuales sirvieron como base para el análisis cuantitativo.

\section{Resultados y discusión}

Desde el análisis de las 366 unidades periodísticas relacionadas con el asesinato del periodista Javier Valdez, durante los dos meses posteriores a su muerte, se han obtenido los siguientes resultados.

Tabla 1. Noticias analizadas por medio y por fecha

\begin{tabular}{|l|r|r|r|r|}
\hline \multicolumn{1}{|c|}{ Diario } & $\begin{array}{c}\text { Mayo } \\
(\mathbf{1 5 - 3 1 )}\end{array}$ & $\begin{array}{r}\text { Junio } \\
\mathbf{( 1 - 3 0 )}\end{array}$ & $\begin{array}{c}\text { Julio } \\
\mathbf{( 1 - 1 5 )}\end{array}$ & Total \\
\hline El Debate & 60 & 13 & 4 & 77 \\
\hline El Universal & 57 & 18 & 6 & 81 \\
\hline Noroeste & 94 & 44 & 12 & 150 \\
\hline Reforma & 48 & 7 & 3 & 58 \\
\hline Total & 259 & 82 & 25 & 366 \\
\hline
\end{tabular}

Fuente: elaboración propia

6 Se agradece a Diana Payan y Harumi Shimizu por su valiosa labor como codificadores 
Los datos cuantitativos obtenidos muestran que el periódico con mayor cobertura y seguimiento noticioso es Noroeste, con un total de 150 notas publicadas sobre el asesinato de Javier Valdez hasta el mes de julio del 2017. Por contraste, el periódico que menos noticias tiene es Reforma. Estos datos no son sorprendentes siendo que el primero es local y el segundo nacional. Sin embargo, sí es llamativo que el periódico nacional El Universal, tiene más notas que el local El Debate.

También permiten comprobar el ciclo de la noticia, la cual inicia con mucha fuerza informativa ya que los primeros quince días se publican el $70.7 \%$ de todas las 366 noticias, después decae notablemente la publicación de notas en todos los periódicos, a tal grado que los últimos quince días se publican solo el $10 \%$ del total.
Para dar respuesta al objetivo de investigación de este artículo se considera oportuno presentar un análisis comparativo de los periódicos por cada una de las categorías y después se muestra el resultado consolidado.

Por lo que se refiere a la primera categoría -de dar contexto sobre los hechos que se describen (BKGD)- los resultados obtenidos muestran que en todos los periódicos se obtuvo un porcentaje a favor war journalism, ya que en ningún caso las noticias que daban contexto, superan el $50 \%$. El periódico nacional Reforma, es el único que tuvo un porcentaje proporcional en cuanto a dar o no dar contexto. Ninguno de los periódicos locales dio un amplio contexto al hecho noticioso, por lo tanto, no se comprueba la hipótesis de partida de que los medios locales darían más contexto que los nacionales (Tabla 2).

Tabla 2. Antecedentes y Contexto

\begin{tabular}{|c|c|c|c|c|}
\hline & Reforma & EI Universal & El Debate & Noroeste \\
\hline $\begin{array}{l}\text { La nota da } \\
\text { contexto: }\end{array}$ & $\begin{array}{l}\text { No - } 50 \% \\
\text { Sí } \\
\text { Cómo se formó la } \\
\text { situación - } 1.7 \% \\
\text { Entender la historia - } \\
5.2 \% \\
\text { Ya estaba } \\
\text { amenazado - } 8.6 \% \\
\text { Lo difícil que es } \\
\text { ejercer el periodismo } \\
\text { - } 34.5 \%\end{array}$ & $\begin{array}{l}\text { No - } 67.9 \% \\
\text { Sí } \\
\text { Crimen } \\
\text { organizado } \\
-1.2 \% \\
\text { Ya estaba } \\
\text { amenazado } \\
\text { - 1.2\% } \\
\text { Lo difícil que es } \\
\text { ejercer el } \\
\text { periodismo } \\
\text { - 29.6\% }\end{array}$ & $\begin{array}{l}\text { No - } 59.7 \% \\
\text { Sí } \\
\text { Ejercicio del } \\
\text { periodismo } \\
\text { - 3.9\% } \\
\text { Ya estaba } \\
\text { amenazado } \\
\text { - 3.9\% } \\
\text { Lo difícil que } \\
\text { es ejercer el } \\
\text { periodismo } \\
\text { - } 32.5 \%\end{array}$ & $\begin{array}{l}\text { No - } 52.7 \% \\
\text { Sí } \\
\text { Cómo se formó la } \\
\text { situación - } 6 \% \\
\text { Entender la historia } \\
\text { - 16.7\% } \\
\text { Ya estaba } \\
\text { amenazado } 2 \% \\
\text { Lo difícil que es } \\
\text { ejercer el periodismo } \\
\text { - 22.7\% }\end{array}$ \\
\hline
\end{tabular}


No obstante, el dato anterior, resulta muy interesante el comprobar que, en todos los periódicos, tanto nacionales como locales, al dar un contexto, se refieren -en un alto porcentaje- a lo difícil que es ejercer el periodismo en México. El dato anterior muestra que los profesionales de la información son conscientes de los peligros que experimentan y quieren hacerlo ver al público. Como se dijo antes, este país es el tercero más peligroso para ejercer el periodismo en el mundo y así debe ser percibido.

En cuanto a la segunda categoría, que hace referencia a las soluciones que presentan los medios (IDEAS) se observa que tres periódicos no presentan soluciones relativas a esclarecer la verdad en torno al asesinato del periodista y al proceso judicial (Tabla 3). Este enfoque es claramente orientado al war journalism. Los resultados no sorprenden porque en México la impunidad ante los crímenes y la corrupción -según el Barómetro Global de la Corrupción el país del sol azteca encabeza la lista en América Latina- son dos lacras que dificultan la vida democrática y el estado de Derecho.

Tabla 3. Ideas/Soluciones

\begin{tabular}{|l|l|l|l|l|}
\hline Categoría & \multicolumn{1}{|c|}{ Reforma } & El Universal & \multicolumn{1}{|c|}{ El Debate } & \multicolumn{1}{c|}{ Noroeste } \\
\hline Hablan de & Sí - 84.\% & Sí - 9.9\% & Sí - 5.2\% & Sí - 26.7\% \\
soluciones: & No - 15.5\% & No - 90.1\% & No - 94.8\% & No - 73.3\% \\
\hline
\end{tabular}

Fuente y elaboración: propia

La tercera categoría analizada es la proactividad de la sociedad civil (PROP) y para determinarla se estableció como indicador el hecho de que las noticias se centraran en protestas o marchas en las que participara la sociedad civil. Para determinar su porcentaje se comparó contra el total de las notas publicadas por cada periódico. Lo primero que llama la atención es la gran dife- rencia que existe en la cobertura de las marchas en los dos periódicos locales: El Debate centra sus notas en las marchas en un $70 \%$, en un sentido inverso proporcional Noroeste lo hace en un $30.7 \%$ (Tabla 4). En el caso de los diarios nacionales encontramos que $\mathrm{El}$ Universal es más proclive a cubrir estás notas, incluso más que el diario local $E l$ Debate

Tabla 4: Proactividad de la sociedad civil

\begin{tabular}{|l|l|l|l|l|}
\hline Categoría & Reforma & El Universal & El Debate & Noroeste \\
\hline Las notas se centran en las & Sí $-19 \%$ & Sí $-42.9 \%$ & Sí $-70.1 \%$ & Sí $-30.7 \%$ \\
acciones de los ciudadanos & No $-81 \%$ & No $-57.1 \%$ & No $-29.9 \%$ & No $-69.3 \%$ \\
\hline
\end{tabular}

Fuente y elaboración: propia 
Por lo que se refiere a las siguientes categorías de análisis, estas se centran en la forma en cómo está escrita la nota, es decir el uso del lenguaje es emotivo o racional (EMOT) y la calificación o no de los actores del hecho en buenos y malos (LABEL). Los resultados obtenidos fueron muy interesantes. Es llamativo que ningún medio cumple con ambas características con un enfoque hacia el peace journalism, es decir que cuando un periódico utiliza un lenguaje más racional, lo propio del peace journalism, por contra sí califica a las partes en buenos y malos lo que indica que es un enfoque de war journalism como les ocurre al Noroeste. También ocurre lo contrario si no califica a las partes (peace journalism) en cambio sí se utiliza un lenguaje más emotivo (war journalism) como hacen El Debate y El Universal. El periódico más equilibrado es Reforma, con porcentajes más similares en ambas variables.

Tabla 5. Lenguaje

\begin{tabular}{|l|l|l|l|l|}
\hline Categoría & \multicolumn{1}{|c|}{ Reforma } & \multicolumn{1}{c|}{ El Universal } & \multicolumn{1}{c|}{ El Debate } & \multicolumn{1}{c|}{ Noroeste } \\
\hline ¿Lenguaje emotivo & Emotivo - 46.6\% & Emotivo - 93.8\% & Emotivo - 76.6\% & Emotivo - 38\% \\
o racional? & Racional - 53.4\% & Racional - 6.2\% & Racional - 23.4\% & Racional - 62\% \\
\hline Calificación de & Sí - 58.6\% & Sí - 8.6\% & Sí -40.3\% & Sí - 56\% \\
buenos y malos & No $-41.4 \%$ & No -91.4\% & No 59.7\% & No - 44\% \\
\hline
\end{tabular}

Fuente y elaboración: propia

\section{Conclusiones}

Los resultados consolidados de las cinco categorías de análisis nos muestran que en general la cobertura del asesinato de Javier Valdez fue con un enfoque hacia el war journalism. Solo en seis de las veinte categorías, correspondientes a los 4 diarios, se aprecia un predominio del enfoque peace journalism -en negrita-. El periódico Reforma tuvo un mayor enfoque al peace journalism.

El año 2017 ha sido un periodo especialmente difícil para los periodistas que trabajan en México. El crimen organizado ha asesinado a 12 periodistas en el ejercicio de su profesión, en su labor diaria de mostrar y denunciar los hechos delictivos que implica el mundo de la droga. No es de extrañar que esta guerra se observe en las coberturas pe- riodísticas y que por lo tanto el resultado obtenido en esta investigación muestre una tendencia marcada al war journalism. En estudios anteriores, donde he comparado periódicos nacionales e internacionales, el resultado mostraba que eran los medios extranjeros los que presentaban un enfoque hacia el peace journalism y quizá esto es así porque hay que tener distancia de los hechos para no narrarlos con pasión y dolor.

Los resultados obtenidos, muestran que todos los periódicos analizados -nacionales y locales- se refieren a lo difícil que es ejercer el periodismo en México. Es importante señalar que estos resultados se dan en un contexto nacional de un malestar latente en la sociedad mexicana por las dificultades que experimenta el ejercicio del periodismo en el país. 
Tabla 6. Tendencias del enfoque en la Cobertura

\begin{tabular}{|c|c|c|c|c|}
\hline Tendencias & Reforma & EI Universal & El Debate & Noroeste \\
\hline & $\begin{array}{l}\text { Enfoque de Peace } \\
\text { journalism } 50 \% \\
\text { Enfoque de War } \\
\text { journalism } 50 \%\end{array}$ & $\begin{array}{l}\text { Enfoque de War } \\
\text { journalism } 67.9 \% \\
\text { Enfoque de Peace } \\
\text { journalism } 32.1 \%\end{array}$ & $\begin{array}{l}\text { Enfoque de War } \\
\text { journalism - } 59.7 \% \\
\text { Enfoque de Peace } \\
\text { journalism } 40.3 \%\end{array}$ & $\begin{array}{l}\text { Enfoque de War } \\
\text { journalism - } 52.7 \% \\
\text { Enfoque de Peace } \\
\text { journalism } 47.3 \%\end{array}$ \\
\hline $\begin{array}{l}\mathrm{Hal} \\
\text { sol }\end{array}$ & $\begin{array}{l}\text { Enfoque de Peace } \\
\text { journalism } 84 \% \\
\text { Enfoque de War } \\
\text { journalism } 15.5 \% \\
\end{array}$ & $\begin{array}{l}\text { Enfoque de War } \\
\text { journalism - } 90.1 \% \\
\text { Enfoque de Peace } \\
\text { journalism } 9.9 \% \\
\end{array}$ & $\begin{array}{l}\text { Enfoque de War } \\
\text { journalism - } 94.8 \% \\
\text { Enfoque de Peace } \\
\text { journalism } 5.2 \% \\
\end{array}$ & $\begin{array}{l}\text { Enfoque de War } \\
\text { journalism - } 94.8 \% \\
\text { Enfoque de Peace } \\
\text { journalism } 26.7 \% \\
\end{array}$ \\
\hline $\begin{array}{l}\text { Las notas } \\
\text { se centran } \\
\text { en las } \\
\text { acciones de } \\
\text { los } \\
\text { ciudadanos }\end{array}$ & $\begin{array}{l}\text { Enfoque de War } \\
\text { journalism } 81 \% \\
\text { Enfoque de Peace } \\
\text { journalism } 19 \%\end{array}$ & $\begin{array}{l}\text { Enfoque de War } \\
\text { journalism - } 57.1 \% \\
\text { Enfoque de Peace } \\
\text { journalism } 42.9 \%\end{array}$ & $\begin{array}{l}\text { Enfoque de Peace } \\
\text { journalism } 70.1 \% \\
\text { Enfoque de War } \\
\text { journalism } 29.9 \%\end{array}$ & $\begin{array}{l}\text { Enfoque de War } \\
\text { journalism } 69.3 \% \\
\text { Enfoque de Peace } \\
\text { journalism } 30.7 \%\end{array}$ \\
\hline $\begin{array}{l}\text { Ler } \\
\text { em } \\
\text { rac }\end{array}$ & $\begin{array}{l}\text { Enfoque de Peace } \\
\text { journalism } 53.4 \% \\
\text { Enfoque de War } \\
\text { journalism } 46.6 \%\end{array}$ & $\begin{array}{l}\text { Enfoque de War } \\
\text { journalism } 93.8 \% \\
\\
\text { Enfoque de Peace } \\
\text { journalism } 6.2 \%\end{array}$ & $\begin{array}{l}\text { Enfoque de War } \\
\text { journalism } 76.6 \% \\
\text { Enfoque de Peace } \\
\text { journalism } 23.4 \%\end{array}$ & $\begin{array}{l}\text { Enfoque de Peace } \\
\text { journalism } 62 \% \\
\text { Enfoque de War } \\
\text { journalism } 38 \%\end{array}$ \\
\hline $\begin{array}{l}\text { Calificación } \\
\text { de buenos y } \\
\text { malos }\end{array}$ & $\begin{array}{l}\text { Enfoque de War } \\
\text { journalism } 58.6 \% \\
\\
\text { Enfoque de Peace } \\
\text { journalism } 41.4 \%\end{array}$ & $\begin{array}{l}\text { Enfoque de Peace } \\
\text { journalism } 91.4 \% \\
\text { Enfoque de War } \\
\text { journalism } 8.6 \%\end{array}$ & $\begin{array}{l}\text { Enfoque de Peace } \\
\text { journalism } 59.7 \% \\
\\
\text { Enfoque de War } \\
\text { journalism } 40.3 \%\end{array}$ & $\begin{array}{l}\text { Enfoque de War } \\
\text { journalism } 56 \% \\
\text { Enfoque de Peace } \\
\text { journalism } 44 \%\end{array}$ \\
\hline
\end{tabular}

Fuente y elaboración propia

Si bien este artículo se centra en un único caso, el asesinato de Javier Valdez, este ha resultado emblemático porque se acribilló en pleno día a un periodista de consolidada trayectoria profesional, amenazado por los narcos y que ejercía el periodismo en uno de los estados más peligrosos de la república mexicana. Posteriores estudios se deben seguir haciendo sobre la cobertura periodística que se ha dado a la muerte de otros profesionales de la información. También se sugiere que otros estudios ahonden en el papel que la opinión pública mexicana debe ejercer sobre los políticos para lograr que los asesinatos de periodistas no queden impunes y para que se proteja más a los profesionales de la información. 


\section{Bibliografía}

Ávila-Zesatti, C. (2014). México en el laberinto de la contradicción: pacificar un país que (oficialmente) no está en guerra. Mexico: Texere.

Berelson, B. (1952). Content analysis in communication research. New York: Free Press.

Bojórquez, I. (2017, mayo 21). Ellos creen que mataron a Javier. Ríodoce. Recuperado de http://riodoce.mx/noticias/columnas/altares-y-sotanos/ellos-creen-que-mataron-a-javier

Committee to Protect Journalists (2017a). Attack on the Press. 2017 Edition. Recuperado de https://cpj.org/attacks/

Committee to Protect Journalists (2017b). Journalists killed in Mexico since 1992. Recuperado de https://cpj.org/americas/mexico/

Committee to Protect Journalists. (2016). Journalists Killed in Mexico/Motive Confirmed. Recuperado de https://cpj.org/killed/americas/mexico/

Cottle, J. (2016). Reporting Fear, Promoting Peace: A role for peace journalism in Mexico. Retrieved October 8, 2017, from https://www.policy-shift.com/single-post/2016/09/15/ Reporting-Fear-Promoting-Peace-A-role-for-peace-journalism-in-Mexico

Galtung, J. and Vincent, R. C. (1992). Global Glasnost: Toward a New World Information and Communication Order. Cresskill, NJ: Hampton Press.

Galtung, J. (1997). The Peace Journalism Option. Taplow

Galtung, J. (1998a). High Road, Low Road Charting the Course for Peace journalism. Track Two: 7 (4), pp. 7-10:

Galtung, J. (1998b). Tras la violencia, 3R: reconstrucción, reconciliación, resolución: afrontando los efectos visibles e invisibles de la guerra y la violencia. Bilbao: Bakeaz.

Galtung, J. (2002). Peace journalism- A Challenge. In: K. Wilhem and H. Luostarinen. Journalism and the New World Order, eds. Göteborg: Nordicom.

Galtung, J. (2007). Peace Journalism as Ethical Challenge. Asteriskos, 3(4), pp. 7-16.

Galtung, J. (2006). Peace Journalism as an Ethical Challenge. Global Media Journal: Mediterranean Edition, 1(2), pp. 1-5.

Gavra, D. P. and Savitskaya, A. S. (2011). Mass media in interstate conflicts: Typological model "peace-conflict journalism multidimensional approach. Russian Journal of Communication, 4(3), pp. 251-265.

Redacción Gatopardo (2017). Periodistas asesinados en México en 2017Al 19 de diciembre, van 12 periodistas asesinados en México en 2017. Las agresiones contra la prensa no se detienen. Gatopardo, 187. Recuperado de https:/www.gatopardo.com/opinion/actualidad/ periodistas-asesinados-en-mexico-en-2017/

Gutiérrez Atala, F., Odriozola Chéné, J. y Domínguez Panamá, J. J. (2017). La satisfacción de los periodistas de Ecuador, Chile y México frente a la formación universitaria y sus implicancias en el ejercicio profesional. Revista de Comunicación, XVI (1), 76-96.

Hostil O. R. (1969). Content analysis for the social sciences and humanities. Addison Wesley.

Krippendorff, K. (1990). Metodología de análisis de contenido. Teoría y Práctica. México: Piados Comunicación. 
Kuusik, N. (2010). The Role of the Media in Peace Building, Conflict Management, and Prevention. E-International Relations, 2010. Recuperado de http://www.e-ir.info/2010/08/28/ the-role-of-media-in-peace-building-conflict-management-and-prevention/

Lee, S.T., Maslog, C.C. and Kim, H.S. (2006). Asian Conflicts and the Iraq War: A Comparative Framing Analysis. International Communication Gazette, 68(5-6), pp. 499-518.

Lynch, J. (2013). A Global Standard for Reporting Conflict. New York: Routledge.

López, R. y Moreno, L. (2008, 3 de enero). 2773 ejecuciones en 2007, Milenio, México. Recuperado de: www.milenio.com/index.php/2008/01/03/170874/

Márquez-Ramirez, M. \& Hughes, S. (2016). Los periodistas en México: panorama cuantitativo de sus perfiles demográficos y profesionales. En A. Amado (Ed), El periodismo por los periodistas: Perfiles profesionales en las democracias de América Latina. Uruguay: Konrad Adenauer Stifung, pp. 86-101.

Meneses, M. E. (2011). Periodismo: Desafíos para su libre ejercicio. En: Panorama de la comunicación en México 2011. Desafíos para la calidad y la diversidad. México: AMEDI.

Monsiváis, C. (2003). Señor Presidente, a usted no le da vergüenza su grandeza? En: Scherer García, J., y Monsiváis, C. (eds.). Tiempo de saber: prensa y poder en México. México: Aguilar, pp. 99-339.

Nicolas-Gavilan, M. T. (2011). Framing the news: from political conflict to peace. How the Framing Theory and Political Context Model can enhance the peace journalism model. Journal of Latin American Communication Research, 1(2), 48-61.

Nicolas-Gavilan, M. T. (2012). The Coverage of Drug Trafficking: Peace and War Journalism in American, Mexican, and Spanish Online Newspapers. Journalism and Mass Communication, 2(7), 717-734.

Nicolas-Gavilan, M. T. (2015). El enfoque del conflicto israeli-palestino. España: La fragua.

Ramírez, D. (2010, septiembre, 26). ¿Qué quieren de nosotros? El Universal. Recuperado de http://archivo.eluniversal.com.mx/editoriales/50002.html

Redacción. (2010, septiembre, 19). ¿Qué quieren de nosotros? El Diario de Ciudad Juárez. Recuperado de http://diario.mx/Local/2010-09-19_cfaade06/_que-quieren-de-nosotros/?/ Redacción. (2017, mayo 17). Cumplen amenaza: asesinan a Javier Valdez en Culiacán. La Jornada. Recuperado de http://www.jornada.unam.mx/2017/05/16/politica/002n 1 pol

Reporteros Sin Fronteras. Clasificacion de la libertad de Prensa 2017. Recuperado de: https://rsf.org/es/datos-clasificacion

Ríos, V. (2012). Tendencias y explicaciones al asesinato de periodistas y alcaldes en México, Las bases sociales y políticas del crimen organizado y la violencia en México, SSP, México.

Ríos, V. (2013, agosto, 1). ¿Quién mata periodistas? Nexos. Recuperado de http://www.nexos. com.mx/?p= 15419

Roach, C. (1993). Communication and Culture in war and peace. Newbury Park: Sage Publications. Rodelo, F. D. (2009). Periodismo en entornos violentos: el caso de los periodistas de Culiacán, Sinaloa. Comunicación y Sociedad. Núm. 12, julio-diciembre, pp. 101-118.

Rodríguez Castañeda, R. (1993). Prensa vendida. Los periodistas y presidentes: 40 años de relaciones. México: Grijalbo. 
Salazar, S. (2012). Periodismo y violencia: la producción de subjetividad del riesgo en el norte de México. Global media Journal, vol. 9. pp. 63-80. Recuperado de: http://www.redalyc.org/ pdf/687/68723565005.pdf

Scherer, J. (1986). Los presidentes. México: Grijalbo.

Shinar, D. (2003). Peace process in cultural conflict: The role of the media. Conflict \& Communication, 2, (1) pp. 1-10.

Shinar, D. (2004). Media Peace Discourse: Constraints, Concepts and Building Blocks. Conflict \& Communication, 3 (1-2).

Tumber, H., \& Waisbord, S. (2017). The Routledge Companion to Media and Human Rights. Taylor \& Francis.

UNESCO. (2017). Tendencias mundiales en Libertad de Expresion y Desarrollo de Medios. Informe mundial 2017/2018. Recuperado de https://es.unesco.org/world-media-trends-2017

Wolfsfeld, G. (1997). Media and Political Conflict. New York: Cambridge University Press.

Wolfsfeld, G. (2004). Media and the path to peace. Cambridge: Cambridge University Press. 